\title{
Cooperative Spectrum Sharing in Cognitive Radio Networks: A Game-Theoretic Approach
}

\author{
Haobing Wang ${ }^{1}$, Lin $\mathrm{Gao}^{1}$, Xiaoying Gan ${ }^{1}$, Xinbing Wang ${ }^{1}$, Ekram Hossain ${ }^{2}$ \\ 1. Department of Electronic Engineering, Shanghai Jiao Tong University, China \\ 2. Department of Electrical and Computer Engineering, University of Manitoba, Canada
}

\begin{abstract}
We consider the problem of cooperative spectrum sharing among a primary user and multiple secondary users, where the primary user selects a proper set of secondary users to serve as the cooperative relays for its transmission. In return, the primary user leases portion of channel access time to the selected secondary users for their own transmission. The primary user decides the portion of channel access time it will leave for the selected secondary users (i.e., the cooperative relays), and the cooperative relays decide their power level used to help primary user's transmission in order to achieve proportional access time to the channel. We assume that the primary user and secondary users are rational and selfish, i.e., they only aim at maximizing their own utility. As secondary user's utility is in term of their own transmission rate and the power cost for primary user's transmission, so they will choose a proper power level to meet the tradeoff between transmission rate and power cost. Primary user will choose a proper portion of channel access time for the cooperative relays to attract them to employ higher power level. We formulate the problem as a non-cooperative game between primary user and secondary users, and it is proved to converge to a unique Stackelberg equilibrium. By employing an iterative updating algorithm, we can achieve the unique equilibrium point.
\end{abstract}

\section{INTRODUCTION}

Frequency spectrum, the most scarce resource for wireless communications, may be congested by diverse users and applications in the next generation wireless networks. Besides, the traditional spectrum allocation mechanism results in a large portion of the scarce spectrum remaining unutilized due to the fact that the spectrum utilization varies significantly with time and location. Cognitive radio has been viewed as a novel approach for improving the utilization of spectrum resource. Cognitive radio networks are designed based on the concept of dynamic spectrum sharing where cognitive radio users can opportunistically share the radio spectrum [1]. There have been a flurry of researchers on cognitive radio networks emphasizing the technical aspects of spectrum sharing (e.g., spectrum sensing in physical layer, dynamic spectrum access protocol in MAC layer, etc.). In this paper, we focus on designing a cooperative spectrum sharing mechanism between primary user and secondary users.

Recently, cooperative mechanism has become an interesting topic in cognitive radio research. In [7], the authors proposed the concept of cooperative cognitive radio network (CCRN) wherein a primary user can involve secondary users as the cooperative relays. In return, the secondary users achieve the opportunity to access the wireless channel for their own data transmission. However, the secondary users' cooperative power is set to be fixed, which is not efficient, and the secondary users' own transmission time is not related to its contribution to primary user's transmission, which is not reasonable. Further, the selected secondary users are forced to help the primary user(s) even if they have no desire to access the channel, which is not practical for the selfish secondary users. Based on these observations, we propose a novel cooperative mechanism to overcome these limitations.

The main contributions of the paper are as follows:

- We formulate the cooperative spectrum sharing problem as a game between one primary user and $N$ secondary users, where primary user select a proper set of secondary users to serve as the cooperative relays for its transmission. In return, the primary user leases portion of channel access time to the selected secondary users for their own transmission. The primary user's strategy is to select proper secondary users and to determine the portion of channel access time left for secondary users' transmission. Secondary users' strategy is to choose the power level used to help primary user's transmission.

- We define utility functions of primary and secondary users. Primary user's utility function is related to its transmission rate, and secondary user's utility function is not only related to its transmission rate but also the cost of power. Secondary user's access time is related to the effort it makes when it acts as cooperative relay, i.e. the power level it uses to help primary user's transmission.

- We prove that there is a unique Nash equilibrium in the network, and we also work out the strategies of primary user and secondary users in the Nash equilibrium. We also propose an iterative algorithm to enable the selfish primary user and secondary users to converge to Nash equilibrium from an arbitrary initial configuration.

The remainder of the paper is organized as follows. Related works are reviewed in Section II. In Section III, we introduce our system model and the utility functions for the primary user and the secondary users. The analysis of the game model is presented in Section IV. In Section V, we describe an implementation protocol for the game model and the iterative algorithm. Simulation results are presented in Section VI and Section VII states the conclusion.

\section{RELATED WORK}

Game theory has been considered as an effective and reasonable method to study the behavior of users in cognitive 
networks, where different users compete for the same resource. The problem of spectrum sharing in cognitive radio networks can be formulated as various game models [2]. A single-stage cooperative dynamic spectrum sharing game is presented in [3] to analyze multiuser orthogonal frequency-division multipleaccess systems. Multi-stage dynamic game and auction game are adopted in [4] for spectrum sharing and power allocation. Also, [5] provides a game theoretical overview of dynamic spectrum sharing from several aspects, namely, analysis of network users' behaviors, efficient dynamic distributed design, and optimality analysis.

In a cooperative cognitive radio network, the primary users lease their spectrum to secondary users for a fraction of time and in exchange, they get the cooperative transmission power from the secondary users. Game-theoretic models were used in [6] and [7] to analyze the performance of cooperation in cognitive radio networks.. However, in [6], the authors adopted Decode-and-Forward (DF) as the cooperation protocol, and it is hard to obtain analytical solution for the secondary users' optimal cooperative power. Therefore, the authors proposed a traversal algorithm to obtain the optimal cooperative power for secondary users, the computational cost of which will be intolerable when the number of secondary user is large. In [7], the secondary users' cooperative power is set to be fixed, which is not efficient. Also, there are some limitations in both [6] and [7]: the secondary users' utility does not consider their contribution to the primary users' transmission, which is not rational. Further, the selected secondary users are forced to help the primary users even if they have no desire to access the channel, which is not practical for the selfish secondary users.

In our model, we design a power-correlative cooperative mechanism for cognitive radio networks, and we adopt Amplify-and-Forward (AF) as the cooperation protocol, which will make it feasible to achieve the analytical solution of the secondary users' optimal cooperative power. We also consider the contribution of secondary users on the primary user's transmission. Our model is more practical since the secondary users' utility is positively related to the contribution they make to primary users' transmission. Therefore, the incentive of secondary users for taking part in the cooperation will be increased, which is beneficial for the whole network. Also, it is more fair in the sense that the secondary user who contributes more to the primary user obtains more revenue.

\section{System MOdEL AND UTILITY FUnCTIONS FOR PRIMARY AND SECONDARY USERS}

\section{A. System Model}

We consider one primary user and multiple secondary users operating in the same area, and the primary and secondary users all do their best to transmit data as much as possible. The primary transmitter selects a set of secondary users $S$ as relays in its data transmission, and in return, the selected secondary users can access the channel according to the primary users' authority, which is announced by primary user at the beginning of a transmission slot. The selected secondary users have to choose a power level to cooperate with the primary link, and the access time is related to its power level.

The detail system model is sketched in Fig. 1. In our model, each time slot is generally divided into two parts: a fraction $\alpha$ of the slot is used for primary user's transmission, while the rest $1-\alpha$ unit time of slot is used for secondary users' transmission. In the former fraction of slot, the first $\frac{1}{2} \alpha$ fraction is used for the transmission from primary transmitter (PT) to secondary transmitters (STs), as shown in Fig. 1(a), and the second $\frac{1}{2} \alpha$ fraction is used for STs to transmit the received data to primary receiver (PR), as shown in Fig. 1(b). In the latter fraction of slot, the selected secondary users access the channel in time-division multiplexing access (TDMA) mode, as shown in Fig. 1(c). The access time secondary user $i$ obtains (denoted by $t_{i}$ ) is proportional to the contribution it makes in the cooperative process, which is related to the cooperative power level $P_{i}$ it chooses:

$$
t_{i}=(1-\alpha) \frac{P_{i} G_{i, P} G_{P, i}}{\sum_{j \in S}\left(P_{j} G_{j, P} G_{P, j}\right)}
$$

where $P_{i}$ denotes the power of $S T_{i}$ used for the cooperation process, $G_{P, i}$ denotes the channel gain between PT and $S T_{i}$ and $G_{i, P}$ denotes the channel gain between $S T_{i}$ and PR. We assume that the channel condition between two nodes is invariable in one time slot, and they are modeled as independent Gaussian random variables.

In our model, the amplify-and-forward (AF) cooperation protocol [8] is employed to implement the cooperation between primary user and secondary users.

First, considering the signal-to-noise ratio(SNR) at PR caused by PT's direct transmission, we can have:

$$
\Gamma_{P}=\frac{P_{0} G_{P}}{\sigma^{2}}
$$

where $P_{0}$ denotes the power of PT used for its own transmission, $G_{P}$ denotes the channel gain between PT and PR, and $\sigma^{2}$ is the noise variance.

Then, we consider secondary users' contribution to primary user's transmission. With secondary user $i$ being primary user's relay, we assume that $X$ is the PT's transmission signal, $Y_{i}$ is the received signal at $S T_{i}$, and $Z_{i}$ is the received signal at $P R$ from $S T_{i}$. So we have

$$
\begin{gathered}
Y_{i}=\sqrt{P_{0} G_{P, i}} X+\eta_{P, i} \\
Z_{i}=\sqrt{P_{i} G_{i, P}} \frac{Y_{i}}{\left|Y_{i}\right|}+\eta_{i, P}
\end{gathered}
$$

where $\eta_{P, i}$ and $\eta_{i, P} \sim N\left(0, \sigma^{2}\right)$. Substituting (3) into (4) gives

$$
Z_{i}=\frac{\sqrt{P_{i} G_{i, P}}\left(\sqrt{P_{0} G_{P, i}} X+\eta_{P, i}\right)}{\sqrt{P_{0} G_{P, i}+\sigma^{2}}}+\eta_{i, P} .
$$

Therefore, the relayed SNR of secondary user $i$ can be written as

$$
\Gamma_{i}=\frac{P_{i} P_{0} G_{i, P} G_{P, i}}{\sigma^{2}\left(P_{i} G_{i, P}+P_{0} G_{P, i}+\sigma^{2}\right)} .
$$




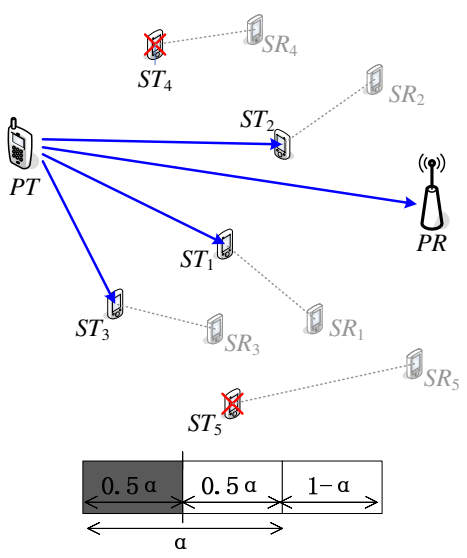

(a)

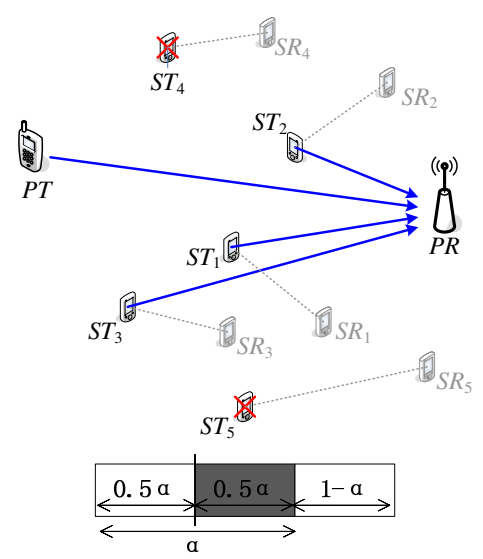

(b)

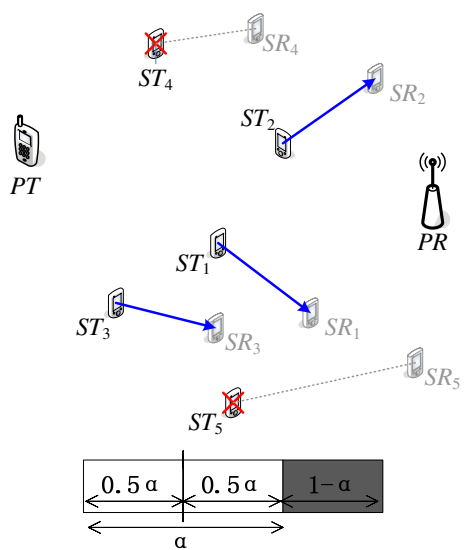

(c)

Fig. 1. System model: (a) in first $\frac{1}{2} \alpha$ fraction of a time slot, PT transmits data to PR and STs; (b) in second $\frac{1}{2} \alpha$ fraction of a time slot, PT and STs transmit data to PR cooperatively; (c) in third $1-\alpha$ fraction of a time slot, secondary users transmit their own data in TDMA mode $(0 \leq \alpha \leq 1)$.

Assuming that the network we discuss is energy constrained, the primary user's transmission rate with relays' help can be presented as follows [9]:

$$
\begin{aligned}
R_{P} & =W \log _{2}\left(1+\Gamma_{P}+\sum_{i \in S} \Gamma_{i}\right) \\
& =W \log _{2}\left(1+\frac{P_{0} G_{P}}{\sigma^{2}}+\sum_{i \in S} \frac{P_{i} P_{0} G_{i, P} G_{P, i}}{\sigma^{2}\left(P_{i} G_{i, P}+P_{0} G_{P, i}+\sigma^{2}\right)}\right) .
\end{aligned}
$$

The transmission rate of secondary user $i$ is given by

$$
R_{i}=W \log _{2}\left(1+\frac{P_{S} G_{i}}{\sigma^{2}}\right), i \in S
$$

where $W$ is the channel bandwidth, $G_{i}$ denotes the channel gain between $S T_{i}$ and $S R_{i}$, and $P_{s}$ denotes the power of secondary users for their own transmission. For simplicity, $W$ will be set to be 1 in the following discussion.

\section{B. Utility Functions}

In this section, we define the utility for both the primary user and the secondary users. Since the primary user's only goal is to maximize its transmission rate, its utility could be defined as follows:

$$
U_{P}=\alpha R_{P}(\alpha)
$$

where $\alpha$ is the portion of a slot primary user retains for its own data transmission. $R_{P}$ is the achievable transmission rate with secondary users' help.

Since the primary user's strategy is to choose a value for $\alpha$ and a set of secondary users $S$ as its relays, $\alpha$ must be set properly. If $\alpha$ is too high, the time remained for secondary users' transmission will be too short. Therefore, secondary users will make less effort in cooperation, i.e. $P_{i}$ will be relatively small. Consequently, the primary transmission rate may decrease. On the other hand, if $\alpha$ is set to be too low, the utility of the primary user will be low. Therefore, an optimization problem for the primary user could be formulated as follows:

$$
\max _{\alpha} U_{P}=\alpha R_{P}(\alpha) \text {, s.t. } 1 \geq \alpha \geq 0 .
$$

A secondary user should consider not only the transmission rate it can achieve, but also the energy cost in helping primary user's transmission. Therefore, a secondary users' goal is to maximize its transmission rate under a reasonable energy cost. Then the utility function of secondary user $i$ could be defined as the difference between its achievable transmission rate and the energy cost in helping primary transmission as follows:

$$
\begin{aligned}
U_{i} & =w_{i} R_{i} t_{i}-\frac{1}{2} \alpha P_{i} \\
& =(1-\alpha) w_{i} \frac{P_{i} G_{i, P} G_{P, i}}{\sum_{j \in S}\left(P_{j} G_{j, P} G_{P, j}\right)} R_{i}-\frac{1}{2} \alpha P_{i}
\end{aligned}
$$

where $w_{i}$ is the equivalent revenue per unit transmission rate for secondary user $i$.

Since a secondary user's strategy is to choose a proper value of $P_{i}$, an optimization problem for $S T_{i}$ can be formulated as follows:

$$
\begin{aligned}
\max _{P_{i}} U_{i} & =(1-\alpha) w_{i} \frac{P_{i} G_{i, P} G_{P, i}}{\sum_{j \in S}\left(P_{j} G_{j, P} G_{P, j}\right)} R_{i}-\frac{1}{2} \alpha P_{i} \\
\text { s.t. } \quad P_{i} \geq 0 . &
\end{aligned}
$$

Note that if any secondary user has no desire to access the channel, it can easily refuse to help the primary user by setting $P_{i}=0$.

\section{Game Theory Analysis}

Based on the utility we define above, we will analyze the game to obtain the equilibrium solutions (i.e., game outcomes). The existence and uniqueness of the equilibrium will be proved. 


\section{A. Optimal Strategies of Secondary Users}

Given the relay set $S$ and slot division parameter by primary user, the selected secondary users' problem is to choose an optimal cooperative power level to maximize its utility. Therefore, a noncooperative power level selection game can be formulated as follows: $G=\left[S,\left\{T_{i}\right\},\left\{U_{i}()\right\}\right]$, where the set of players is the selected relays set $S$, the strategy set of $S T_{i}$ is $T_{i}$, and the utility of $S T_{i}$ is $U_{i}()$. Each secondary user $i$ must select its strategy within the strategy space $\mathbf{T}=\left[T_{i}\right]_{i \in S}$ : $P_{i} \geq 0$ to maximize its utility function $U_{i}\left(P_{i}, \mathbf{P}_{-\mathbf{i}}\right)$.

Theorem 1: A Nash equilibrium exists in the noncooperative power level selection game, $G=\left[S,\left\{T_{i}\right\},\left\{U_{i}()\right\}\right]$.

Due to space limitations, we do not present the detail proof. For details, please refer to our technical report [12].

By Theorem 1, we know that a Nash equilibrium exists in the noncooperative game and $U_{i}(\mathbf{P})$ is concave in $P_{i}$. So we can get the best-response function when the first derivative of $U_{i}$ with respect to $P_{i}$ equals to 0 , i.e.,

$\frac{\partial U_{i}}{\partial P_{i}}=(1-\alpha) w_{i} \frac{G_{i, P} G_{P, i} \sum_{j \in S, j \neq i}\left(P_{j} G_{j, P} G_{P, j}\right)}{\left(\sum_{j \in S}\left(P_{j} G_{j, P} G_{P, j}\right)\right)^{2}} R_{i}-\frac{1}{2} \alpha$ $=0$

for arbitrary selected secondary user $i \in S$. Through simple manipulations, we have

$$
\begin{aligned}
& P_{i}^{*}=\frac{1}{G_{i, P} G_{P, i}}\left(\sqrt{2 w_{i} \frac{1-\alpha}{\alpha} G_{i, P} G_{P, i} A R_{i}}-A\right) \\
& \quad \text { if } \quad A<2 w_{i} \frac{1-\alpha}{\alpha} G_{i, P} G_{P, i} R_{i}
\end{aligned}
$$

where $A=\sum_{j \in S, j \neq i}\left(P_{j} G_{j, P} G_{P, j}\right)$.

Theorem 2: The Nash equilibriun of the noncooperation power level selection game is unique.

For the detailed proof, please see [12].

Then we solve the equation set (13) for each secondary user $i \in S$, we can get the unique Nash equilibrium of the noncooperative power level selection game:

$$
P_{i}^{*}=\frac{2(1-\alpha)\left(N_{0}-1\right)}{\alpha G_{i, P} G_{P, i} B^{2}}\left(B-\frac{N_{0}-1}{w_{i} G_{i, P} G_{P, i} R_{i}}\right)
$$

where $B=\sum_{j \in S} \frac{1}{w_{j} G_{j, P} G_{P, j} R_{j}}$ and $N_{0}$ is the total number of selected secondary users, i.e., $N_{0}=|S|$.

Since the power level can not be negative, we have the following constraints from equation (15):

$$
B>\frac{N_{0}-1}{w_{i} G_{i, P} G_{P, i} R_{i}}, \forall i \in S
$$

which will be used later to select the cooperative relay set.

\section{B. Optimal Strategy of the Primary User}

As mentioned above, the optimization problem for primary user is presented in equation (10). Substitute (15) into $U_{P}$ :

$U_{P}=\alpha \log _{2}\left(1+\frac{P_{0} G_{P}}{\sigma^{2}}+\sum_{i \in S} \frac{P_{i}^{*} P_{0} G_{i, P} G_{P, i}}{\sigma^{2}\left(P_{i}^{*} G_{i, P}+P_{0} G_{P, i}+\sigma^{2}\right)}\right)$,
The primary user's objective is to maximize its utility by selecting optimal parameter $\alpha^{*}$. Therefore, by the first order optimality condition, we have

$$
\frac{\partial U_{P}}{\partial \alpha}=R_{P}(\alpha)+\alpha \frac{\partial R_{P}(\alpha)}{\partial \alpha}=0 .
$$

Substituting (7) into (18), we obtain the optimal $\alpha^{*}$ as follows:

$$
\alpha^{*}=\alpha^{*}\left(\sigma^{2},\left\{w_{i}\right\},\left\{G_{i}\right\},\left\{G_{i, P}\right\},\left\{G_{P, i}\right\}\right), i \in S .
$$

For $0 \leq \alpha^{\prime} \leq 1, U_{p}\left(\alpha^{\prime}\right) \leq U_{p}\left(\alpha^{*}\right)$. For $\quad \forall P_{i}^{\prime} \geq 0, U_{i}\left(P_{1}^{*}, P_{2}^{*}, \cdots, P_{i}^{\prime}, \cdots, P_{N_{0}^{*}}\right) \leq$ $U_{i}\left(P_{1}^{*}, P_{2}^{*}, \cdots, P_{i}^{*}, \cdots, P_{N_{0}^{*}}\right)$. Therefore, we finally obtain the optimal parameter $\alpha^{*}$ and the corresponding optimal cooperative power level $P_{i}^{*}$. The solutions (15) and (19) form a Stackelberg equilibrium, which will be proved in the following subsection.

\section{Proof of the Equilibrium}

In this subsection, we will prove that the solutions (15) and (19) form a Stackelberg equilibrium.

Property 1: For $\forall i \in S$, the optimal power level $P_{i}^{*}$ is decreasing with $\alpha$.

Property 2: Primary user's utility $U_{P}$ is concave in $\alpha$.

Theorem 3: $P_{i}(i \in S)$ and $\alpha$ given by (15) and (19) is a Stackelberg equilibrium for the model in this paper.

For the detailed proof, please see [12].

\section{Implementation of the CoOperative Game MODEL}

In this section, we will propose a cooperation protocol which can be implemented in a practical system. We assume that the channels are stable and the channel condition are detected by corresponding terminal: $G_{i, P}$ is detected by PR, $G_{P, i}$ is detected by $S T_{i}$ and $G_{i}$ is detected by $S R_{i}$. Then the channel condition and other necessary parameter will be sent to PT. Based on these information, the primary user can select a proper set of secondary users as its cooperative relays and calculate the optimal portion parameter $\alpha^{*}$. At the beginning of transmission, the primary user will set $\alpha$ to be 1 , then it will decrease $\alpha$ gradually until $U_{P}$ achives its maximal value, i.e., $\alpha$ reaches its optimal value. Also, primary user will broadcast the value of $\sum_{j \in S} \frac{1}{w_{j} G_{j, P} G_{P, j} R_{j}}$ to secondary users, so that secondary users can calculate their optimal power level corresponding to received value of $\alpha$. In the following subsections, we will discuss the relay selection method and the updating algorithm.

\section{A. Relay Selection}

Since all the analysis above is based on the assumption that the relay set $S$ has already been selected, we will discuss the relay selection method in this subsection.

, In order to accomodate the possible users in good position in the network, we select secondary users who satisfies the selection criteria (16). Without loss of generality, we assume that $w_{1} G_{1, P} G_{P, 1} R_{1} \geq w_{2} G_{2, P} G_{P, 2} R_{2} \geq \ldots \geq$ 
$w_{N_{S}} G_{N_{S}, P} G_{P, N_{S}} R_{N_{S}}$, where $N_{S}$ is the number of secondary users in the network. Then we will select the secondary users as relays following the algorithm shown in Table A.

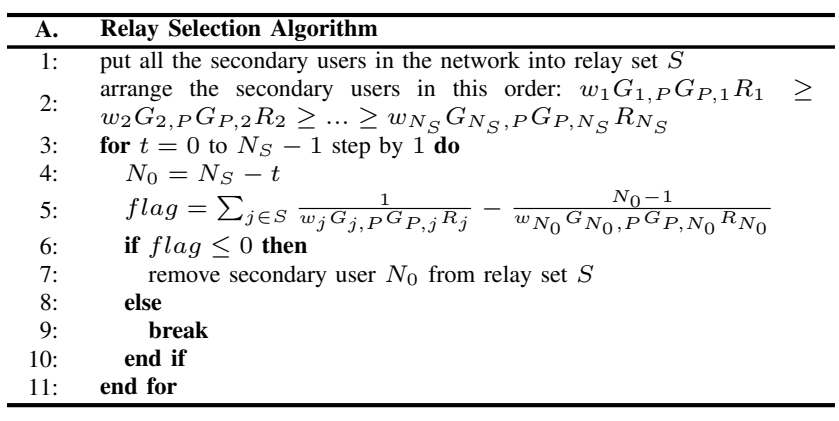

Theorem 4: Assume that there exists a possible relay set $S_{0}=\left\{i \mid 1 \leq i \leq N_{R}\right\}$, i.e., secondary user $i\left(1 \leq i \leq N_{R}\right)$ is in $S_{0}$ and satisfies the selection criteria (16). For $\forall S^{\prime} \subseteq S_{0}$, $S^{\prime}$ is also a possible relay set.

It is shown by Theorem 4 that the algorithm in Table A is an effective way to select possible secondary users in good position as relay.

\section{B. Iterative Algorithm for Primary User}

We propose an updating algorithm to achieve the Stackelberg equilibrium. As the selected secondary users must adapt their strategy according to primary user's strategy, the primary user can be regarded as the "leader" and the selected secondary users can be regarded as the "follower". We only need to design an updating algorithm for the primary user to achieve its optimal parameter $\alpha^{*}$. Then, according to Theorem 3, each selected secondary user will achieve its own optimal power level $P_{i}^{*}$.

By Property 2, we know that the primary user's utility $U_{P}$ is concave in $\alpha$. Therefore, we set $\alpha$ to be 1 at the beginning, and then decrease $\alpha$ gradually. By the concavity of $U_{P}$, we can finally achieve the optimal $\alpha^{*}$. This is reasonable since the primary user retains the entire time slot for its own transmission at the beginning. Then, the primary user will increase the time left for the secondary users' transmission. Correspondingly, the selected secondary users will increase their cooperative power level $P_{i}$, which will increase the primary user's transmission rate. In this way, the primary user will maximize its utility.

We define the updating algorithm as follows: at the beginning, we set $\alpha$ to be 1 . In each loop, the primary user calculates its utility according to current value of $\alpha$ and record it, and compares its utility in the previous loop. If the primary user's utility is still increasing, i.e., we haven't achieved the optimal $\alpha^{*}$ yet, $\alpha$ will be lower by a very small value $\delta$. If the primary user's utility is not increasing, which means we have achieved the optimal $\alpha^{*}$, we should set $\alpha$ to be $\alpha+\delta$, which is the optimal $\alpha^{*}$. Then, we stop updating $\alpha$. The entire updating process is presented in Table B.

It is obvious that we can finally achieve a value which lies in the interval $\left(\alpha^{*}-\delta, \alpha^{*}+\delta\right)$. When $\delta$ is small enough, we can approximate the achieved value as $\alpha^{*}$.

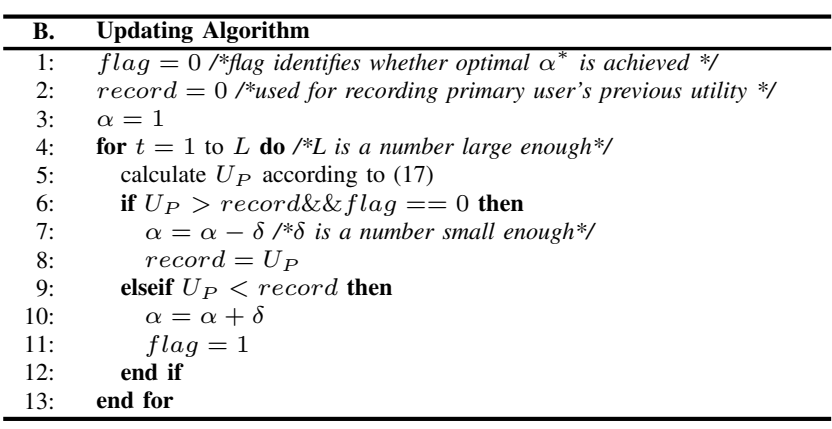

\section{Simulation Results And Analysis}

In this subsection, we consider a geometrical model where PT is located at coordination $(0,0)$, PR is located at coordination $(1,0)$, and secondary tranmitters are located randomly on a square centering on $(0.5,0)$ with side length $D=1$. The secondary receivers are located randomly on a unit square centering on corresponding secondary transmitter. The propagation loss factor is set to be 2 . There are 10 secondary users supposed to exist in the network, and $w_{i}$ are all set to be 10. The noise level is $\sigma^{2}=10^{-4}$ and updating step $\delta=10^{-5}$.

Fig. 2 presents the topology of the simulating network. The blue circles and green squares represent STs and SRs, respectively, and a pair of ST and SR is connected by a green line. The STs, which are selected by PT as cooperative relays, are identified by solid circles; while other STs are identified by hollow circles. Without loss of generality, we mark the secondary users according to the order: $w_{1} G_{1, P} G_{P, 1} R_{1} \geq$ $w_{2} G_{2, P} G_{P, 2} R_{2} \geq \cdots \geq w_{10} G_{10, P} G_{P, 10} R_{10}$.

It is easy to see from Fig. 2 that the first 6 secondary users are selected by the PT as cooperative relays. There are two factors that affect the relay selection: location of ST and the distance between ST and SR. The location of $S T_{i}$ will affects the channel condition between $S T_{i}$ and primary users, i.e., the value of $G_{P, i}$ and $G_{i, P}$. As a secondary user with higher $G_{P, i}$ and $G_{i, P}$ will better help primary user's transmission, so it is beneficial for PT to select such secondary users as relays. Also, the distance between $S T_{i}$ and $S R_{i}$ will affect the value of $G_{i}$. Since a secondary user with higher $G_{i}$ will obtain more revenue from the cooperation with the same power cost, such a secondary user will have more incentive to take part in cooperation, which is beneficial for the primary user. For this reason, all the selected secondary users in Fig. 2 are in good position and the distance between transmitter and receiver are not too long.

Fig. 3 presents the utility of primary user when $\alpha$ varies from 0 to 1 . We find that $\alpha *=0.96$ and the corresponding optimal primary user's utility $U_{P}\left(\alpha^{*}\right)=16.43$. When $\alpha=1$, i.e., the primary user retains the entire time slot for its own transmission, $U_{P}(1)=13.2879$. Since the secondary users will have no incentive to take part in the cooperation, $U_{P}(1)$ will be less than $U_{P}\left(\alpha^{*}\right)$. According to simulation results, the improvement on primary user's utility is $20 \% \sim 35 \%$. Therefore, it is beneficial to implement the cooperation with parameter $\alpha=\alpha^{*}$. 


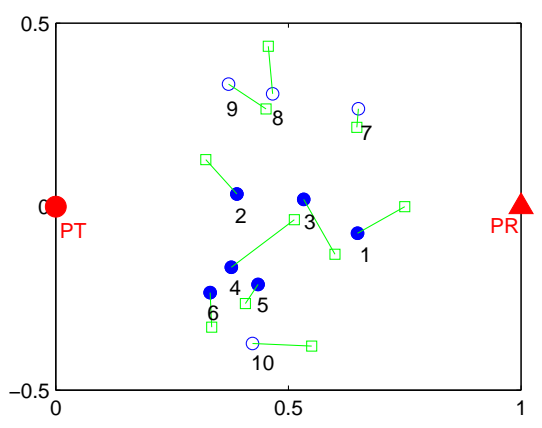

Fig. 2. Topology of the system

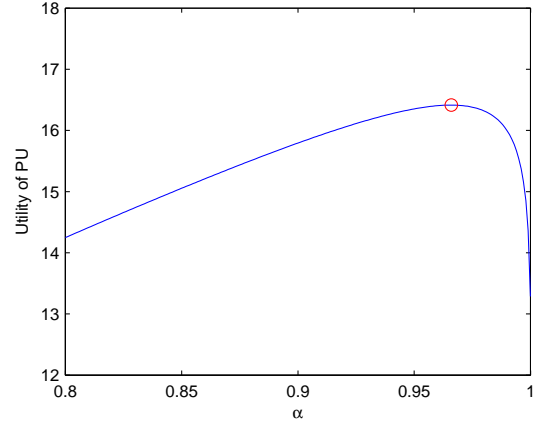

Fig. 3. Optimal $\alpha$ of primary user
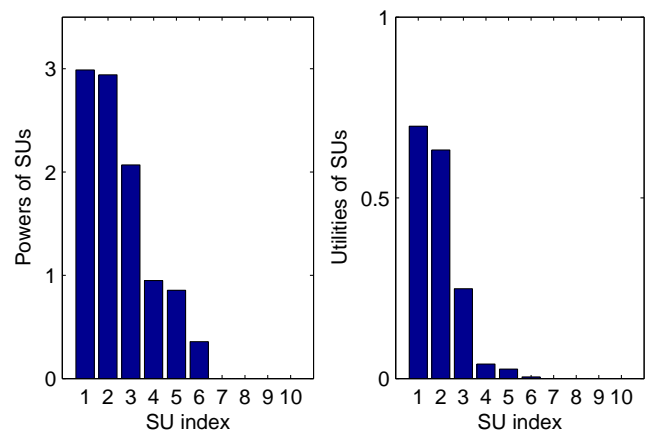

Fig. 4. Secondary users' optimal power and utility
To study the behavior of secondary users, we focus on the cooperative power they choose and the revenue they get. In Fig. 4, the secondary user with better channel condition offers higher cooperative power and get more revenue. According to (1) and (9), the secondary user with better channel condition will get more revenue from the cooperation with the same power cost, so it will tend to offer higher cooperative power. In return, owing to the higher cooperative power and higher channel gain, the secondary user with good channel condition will get more revenue, i.e., higher utility. It is also reasonable that the secondary users who are not selected offers no power and their utility is 0 .

Furthermore, we investigate the impact on the utility of PT in different relay selection on the system. We also simulate the NE in different system topology. For the details, please refer to [12].

\section{CONCLUSION}

We have proposed a novel cooperation mechanism in a cognitive radio network, where primary user select proper secondary users as cooperative relay. In return, the primary user leases portion of a time slot to secondary users for their transmission. In the proposed mechanism, secondary users' access opportunity is positively correlated to its cooperative power level, which motivates the cooperation. We are able to prove that a unique Nash equilibrium exists. We have proposed an iterative algorithm to achieve the Nash equilibrium. Also, we have proposed a relay selection method to accomodate all the possible users in the network. Numerical results have shown that the iterative approach is feasible, and the primary user will select the secondary users at good locations as its relays and the secondary users with better channel condition will achieve higher utility.

\section{REFERENCES}

[1] E. Hossain, D. Niyato, and Z. Han, Dynamic Spectrum Access and Management in Cognitive Radio Networks, Cambridge University Press, 2009.

[2] M. Felegyhazi and J. P. Hubaux, "Game Theory in Wireless Networks: A Tutorial," technical report, LCA-REPORT-2006-002, February, 2006.

[3] Z. Han, Z. Ji, and K. Liu, "Fair Multiuser Channel Allocation for OFDMA Networks Using Nash Bargaining Solutions and Coalitions," IEEE Trans. on Сотти., 53(8): 1366-1376, Aug. 2005.
[4] J. Huang, R. Berry, and M. Honig, "Auction-based Spectrum Sharing," Mobile Networks and Applications, 11(3):405-408, June 2006.

[5] Z. Ji and K. Liu, "Cognitive Radios for Dynamic Spectrum Access \& Dynamic Spectrum Sharing: A Game Theoretical Overview," IEEE Communications Magazine, 45(5):88-94, May 2007.

[6] I. Stanojev, O. Simeone, Y. Bar-Ness et al., "Spectrum Leasing via Distributed Cooperation in Cognitive radio," in Proc. IEEE ICC'08, 2008.

[7] J. Zhang and Q. Zhang, "Stackelberg Game for Utility-Based Cooperative Cognitive Radio Networks," in Proc. ACM Mobihoc '09, 2009.

[8] J. Laneman, D. Tse, and G. Wornell, "Cooperative Diversity in Wireless Networks: Efficient Protocols and Outrage Behavior," IEEE Trans. on Information Theory, vol. 50, no. 12, pp. 3062-3080, Dec 2004

[9] B. Wang, Z. Han, and K. J. Ray Liu, "Distributed Relay Selection and Power Control for Multiuser Cooperative Communication Networks Using Buyer/Seller Game," in Proc. IEEE Infocom '07, 2007.

[10] G.Debreu, "A Social Equilibrium Existence Theorem," in Proc. Nat. Acad. Science, 38.

[11] R.Yates, "A Framework for Uplink Power Control in Cellular Radio Systems," IEEE J. on Sel. Areas in Coтmu., 13(7):1341-1347, Sept. 1995.

[12] H. Wang, "A Novel Cooperative Mechanism for Cognitive Radio Networks," Technique Report, http://iwct.sjtu.edu.cn/Personal/xwang8/cocr.pdf 